\title{
Safety and tolerability of subcutaneous immunoglobulin $20 \%$ in primary immunodeficiency diseases from two continents
}

\author{
Daniel Suez ${ }^{1}$, Gergely Kriván², Stephen Jolles ${ }^{3}$, Mark Stein ${ }^{4}$, Sudhir Gupta ${ }^{5}$, Kenneth \\ Paris $^{6}$, P Martin van Hagen ${ }^{7}$, Nicholas Brodszki ${ }^{8}$, Werner Engl ${ }^{9}$, Heinz Leibl ${ }^{10}$, Barbara \\ McCoy $^{10}$ \& Leman Yel*,11,12 \\ ${ }^{1}$ Allergy, Asthma \& Immunology Clinic, PA, Irving, TX 75063, USA \\ ${ }^{2}$ Department of Pediatric Hematology \& Stem Cell Transplantation, Szent László Hospital, Budapest, Hungary \\ ${ }^{3}$ Immunodeficiency Centre for Wales, University Hospital of Wales, Heath Park, Cardiff, UK \\ ${ }^{4}$ Allergy Associates of the Palm Beaches, North Palm Beach, FL 33408, USA at the time of this study \\ ${ }^{5}$ Division of Basic \& Clinical Immunology, University of California at Irvine, Irvine, CA 92697, USA \\ ${ }^{6}$ Department of Pediatrics, LSU Health Sciences Center New Orleans, Children's Hospital of New Orleans, New Orleans, \\ LA 70118, USA \\ ${ }^{7}$ Department of Internal Medicine/Clinical Immunology, Erasmus University Medical Center, Rotterdam, Netherlands \\ ${ }^{8}$ Department of Pediatric Oncology/Immunology, Skåne University Hospital, Lund, Sweden \\ ${ }^{9}$ Biostatistics, The Takeda Group of Companies, Vienna, Austria \\ ${ }^{10} \mathrm{Clinical}$ Research Immunology, The Takeda Group of Companies, Vienna, Austria \\ ${ }^{11}$ Research \& Development, Plasma Derived Therapies Business Unit, The Takeda Group of Companies, Cambridge, \\ MA 02142, USA \\ ${ }^{12}$ Department of Medicine, University of California at Irvine, CA 92697, USA \\ *Author for correspondence: Tel.: + 1617588 8437; leman.yel@takeda.com
}

\begin{abstract}
Aim: This pooled analysis evaluated the safety and tolerability of the subcutaneous immunoglobulin $20 \%$ product, Ig20Gly, in primary immunodeficiency diseases using data from two Phase II/III studies conducted in North America and Europe. Patients \& materials/methods: Patients received Ig20Gly (volumes, $\leq 60 \mathrm{ml} / \mathrm{site}$; rates, $\leq 60 \mathrm{ml} / \mathrm{h} / \mathrm{site}$ ). Adverse events (AEs), tolerability and infusion parameters were assessed. Results: Patients ( $2-83$ years; $N=122$ ) received 6676 Ig20Gly infusions. No causally related serious or severe AEs were reported. Thirty-five patients ( $28.7 \%)$ reported 232 causally related local AEs. Twentyseven patients $(22.1 \%)$ reported 165 causally related systemic AEs. There was no association between the infusion volume or rate and causally related local AEs. Conclusion: Ig20Gly was well tolerated in a broad population of patients with primary immunodeficiency diseases.
\end{abstract}

First draft submitted: 4 April 2019; Accepted for publication: 18 June 2019; Published online:

2 July 2019

Keywords: common variable immune deficiency $\bullet$ CUVITRU $\bullet$ immunoglobulin $\bullet$ immunoglobulin replacement therapy $(\operatorname{IgRT}) \bullet \lg 20 \mathrm{Gly} \bullet$ primary immunodeficiency $\bullet$ safety $\bullet$ subcutaneous $\bullet$ tolerability

There are more than 300 distinct primary immunodeficiency diseases (PID) resulting from more than 320 different gene mutations [1]. Many of these conditions are associated with defective production or low levels of immunoglobulin (Ig) that make patients more susceptible to bacterial and viral infections. Ig replacement therapy is indicated for the treatment of PID with defective antibody production and has been shown to reduce the frequency and severity of infections [2].

Immunoglobulin replacement therapy may be administered by intravenous (IV) or subcutaneous (SC) infusions [2,3], with the clinical response as the primary driver of dosing decisions. IV administration of IG (IVIG) requires monthly doses of $300-800 \mathrm{mg} / \mathrm{kg}$ body weight and is typically administered every 3-4 weeks over 2-4 h. IVIG is delivered rapidly into the systemic circulation, which leads to peaks of serum Ig levels that are thought to be associated with an increased incidence of systemic adverse reactions [3]. In contrast, SC infusion of IG (SCIG) allows 
slow diffusion of IG from the SC tissue into systemic circulation, smoothing significant serum level variations and leading to stable IgG levels and lower rates of systemic adverse events (AEs) compared with IV administration [3]. In addition, SCIG infusion offers the convenience of self-administration at home [4,5]. Drawbacks of SCIG therapy compared with IVIG include a higher incidence of local adverse reactions and a need for more frequent infusions and multiple infusion sites due to the limited volume that can be infused into each site [3,6].

The development of more highly concentrated SCIG products allows for the infusion of the same dose in smaller volumes compared with less concentrated products permitting fewer infusion sites and possibly reducing the frequency of infusions [6]. One such preparation, Ig20Gly (CUVITRU, immune globulin subcutaneous [human] $20 \%$ solution; Baxalta US, Inc., a member of the Takeda group of companies, CA, USA), is a ready-for-use human IgG preparation produced with a manufacturing process similar to the IVIG $10 \%$ solution (GAMMAGARD LIQUID/Kiovig, Baxalta US, Inc., a member of the Takeda group of companies), except for an additional step of ultra-/diafiltration resulting in a final protein concentration of $20 \%$ (weight/volume). Ig20Gly can be infused at up to $60 \mathrm{ml} / \mathrm{h}$ per site and has been shown to be efficacious and well tolerated in two Phase II/III clinical studies conducted in Europe [7] and North America [8]. The safety and tolerability of Ig20Gly were assessed in both trials in a consistent manner facilitating a pooled analysis of AEs and tolerability data from these two studies.

\section{Patients \& methods}

Study design

This is a pooled analysis of two prospective, open-label clinical trials in Europe (ClinicalTrials.gov NCT01412385) [7] and North America (ClinicalTrials.gov NCT01218438) [8]. Both studies were performed in accordance with the Declaration of Helsinki and the international standards of Good Clinical Practice. Patients were enrolled at 15 sites in the USA and Canada for the North American study, and at 16 sites in seven countries (Germany, Austria, Sweden, The Netherlands, Great Britain, Belgium and Hungary) for the European study. Written informed consent was obtained from all patients before undergoing any study procedure. Details of the inclusion and exclusion criteria and study design have been published previously $[7,8]$. In brief, patients were eligible for study inclusion if they were aged $\geq 2$ years with a documented diagnosis of humoral immunodeficiency for which they had been receiving a stable monthly equivalent dose of IG (IV or SC) at an average dose of $300-1000 \mathrm{mg} / \mathrm{kg}$ body weight every 4 weeks for $\geq 3$ months before enrollment and had a serum IgG trough level of $>500 \mathrm{mg} / \mathrm{dl}$ at screening $[7,8]$.

The North American trial consisted of four study periods. During period 1, all patients received IVIG 10\% for 13 weeks to determine the area under the curve for IgG after IVIG treatment. During periods 2-4, patients received Ig20Gly. In period 2, Ig20Gly was administered in some patients at a dose adjusted to $145 \%$ of the IVIG dose for approximately 12-16 weeks to assess bioequivalence; in period 3, all patients received the same adjusted dose for 12 weeks. Patients received Ig20Gly at individually adapted doses during period 4 (40 weeks) [8].

The European trial consisted of two periods. During period 1, patients received IVIG 10\% for 13 weeks or SCIG $16 \%$ for 12 weeks to attain a stable baseline serum IgG before starting Ig20Gly treatment. Patients were treated with Ig20Gly for 52 weeks during period 2 [7].

Patients had the choice to infuse Ig20Gly into one site or multiple sites simultaneously using a T34L electromechanical syringe driver (Caesarea Medical Electronics, Caesarea, Israel) and a high-flow 24-gauge needle set (MarCal Medical, MD, USA) ranging in length from $6-12 \mathrm{~mm}$, at the discretion of the investigator $[7,8]$. Infusion sites were rotated to avoid repeated use of the same site within a short period. Infusion rates were increased incrementally. The first two infusions were initiated at a rate of $10 \mathrm{ml} / \mathrm{h}$ per site and could be increased to a maximum of $20 \mathrm{ml} / \mathrm{h}$ per site. Subsequent infusions could be infused at a maximum rate of $60 \mathrm{ml} / \mathrm{h}$ per site as tolerated. Infusion volumes up to $60 \mathrm{ml}$ per site were permitted in patients with body weight $\geq 40 \mathrm{~kg}$ as tolerated or according to patient preference and physician discretion. For patients weighing $<40 \mathrm{~kg}$, the infusion volume was $\leq 20 \mathrm{ml}$ per site for the initial two infusions and then increased up to $60 \mathrm{ml}$ per site as tolerated. Home infusion was allowed with the assistance of a healthcare professional or by the patient or a caregiver after adequate training $[7,8]$.

\section{Safety assessments}

Safety was assessed by clinical and laboratory assessments throughout the two studies $[7,8]$. AEs occurring during infusions conducted at the study site (the first three infusions, and subsequently every 8-12 weeks) were recorded by the investigator. Investigators were trained on the assessment of potential AEs. Investigators also provided guidance to the patient or caregiver regarding the identification and documentation of local and systemic AEs in the event 
Table 1. Patient demographics.

\begin{tabular}{|c|c|c|c|c|c|c|}
\hline \multirow[t]{2}{*}{ Demographic } & \multicolumn{6}{|c|}{ Age group (years) } \\
\hline & $<6(n=6)$ & $6-11(n=22)$ & $12-15(n=11)$ & $16-64(n=71)$ & $\geq 65(n=12)$ & $\begin{array}{l}\text { All patients } \\
(\mathrm{N}=122)\end{array}$ \\
\hline \multicolumn{7}{|l|}{ Sex, $\mathrm{n}(\%)$} \\
\hline - Male & $5(83.3)$ & $19(86.4)$ & $9(81.8)$ & $34(47.9)$ & $1(8.3)$ & $68(55.7)$ \\
\hline - Female & $1(16.7)$ & $3(13.6)$ & $2(18.2)$ & $37(52.1)$ & $11(91.7)$ & $54(44.3)$ \\
\hline Median (range) age, years & $4(2-5)$ & $8(6-11)$ & $13(12-13)$ & $39(16-63)$ & $67(65-83)$ & $32(2-83)$ \\
\hline Median (range) weight, kg & $15.9(13-39)$ & $29.1(19-56)$ & $50.8(30-82)$ & $76.2(48-162)$ & $67.4(48-113)$ & $66.8(13-162)$ \\
\hline $\begin{array}{l}\text { Mean (SD) Ig20Gly dose, } \\
\mathrm{g} / \mathrm{kg} / \text { week }\end{array}$ & $0.17(0.05)$ & $0.18(0.05)$ & $0.19(0.10)$ & $0.19(0.08)$ & $0.21(0.06)$ & $0.19(0.08)$ \\
\hline
\end{tabular}

that an AE were to occur at home. Patients were instructed to inform the investigator or site immediately if an $\mathrm{AE}$ occurred. Patients were also contacted by the investigator within 3-5 days of each infusion either at the study site or at home as follow-up to ensure appropriate AE documentation. Patients used an eDiary tablet to continuously record home treatments, AEs and additional information. Investigators reviewed patients' eDiary entries at every site visit. All AEs were assessed by the investigator for seriousness, severity, temporal association (occurring within $72 \mathrm{~h}$ of the infusion) and possible causal relatedness to Ig20Gly.

Routine hematology screening was performed to monitor for hemolysis. In the event of a $\geq 2 \mathrm{~g} / \mathrm{dl}$ decrease in hemoglobin, assessments for hemolysis were to be performed within 48-72 h of receipt of the hemoglobin value. Hemolysis assessments included a direct antiglobulin (Coombs) test, plasma-free hemoglobin level, reticulocyte count, lactate dehydrogenase, serum haptoglobin and urine hemosiderin $[7,8]$.

\section{Results}

A total of 122 patients aged 2-83 years received Ig20Gly for a median (range) of 365 (30-629) days in the two clinical trials (Table 1). Overall, 68 male and 54 female patients were enrolled; however, the male to female ratio varied with age. Most patients aged $\leq 15$ years were male $(33 / 39,84.6 \%)$, whereas more patients aged $\geq 16$ years were female $(48 / 83,57.8 \%)$. A total of 6676 Ig20Gly infusions were administered to the 122 patients enrolled in the two trials.

In the pooled population, 112 patients $(91.8 \%)$ completed the planned duration of treatment. In the North American trial, 74 patients received Ig20Gly. Of these, seven patients discontinued: five patients withdrew consent for reasons unrelated to AEs, one patient discontinued owing to an AE unrelated to Ig20Gly and one patient discontinued owing to poor adherence to the study protocol. In the European study, 48 patients received Ig20Gly. Of these, three patients discontinued during Ig20Gly treatment: one patient reported pain during and after administration of Ig20Gly and chose to stop participation, and two patients withdrew consent for reasons unrelated to an $\mathrm{AE}$.

\section{Adverse events}

There were no serious or severe AEs related to Ig20Gly treatment reported in either study. Overall, 397 nonserious AEs that were considered to be causally related to Ig20Gly (frequency rates of 0.059 per infusion) were reported in 48 of 122 patients (39.3\%) enrolled in the two trials.

Thirty-five patients (28.7\%) reported a total of 232 causally related local AEs in $2.7 \%$ of infusions with frequency rates of 0.035 per infusion (Table 2). All Ig20Gly causally related local AEs were considered to be mild (226 AEs; 0.034 /infusion) or moderate ( $6 \mathrm{AEs} ;<0.001 /$ infusion) in severity. The most common local reactions were infusion site erythema (0.012/infusion), infusion site pain (0.009/infusion), and infusion site swelling (0.007/infusion). Of note, $34.1 \%(79 / 232)$ of the local AEs deemed causally related to Ig20Gly were reported by the parent of one patient; all 79 AEs were mild. This patient/parent completed the study and expressed preference for Ig20Gly over other IgG treatment options at the end of the study.

A total of 27 of 122 patients (22.1\%) reported 165 systemic AEs that were deemed causally related to Ig20Gly, with frequency rates of 0.025 per infusion (Table 3). All Ig20Gly causally related systemic AEs were considered to be mild (101 AEs; 0.015/infusion) or moderate (64 AEs; 0.010/infusion) in severity. The most frequently reported causally related systemic AEs were headache (0.010/infusion), diarrhea (0.007/infusion) and fatigue 
Table 2. Causally related ${ }^{\dagger}$ local adverse events (excluding infections) occurring in $\geq 1 \%$ of patients.

\begin{tabular}{|c|c|c|c|c|c|}
\hline Adverse event & AEs, $n$ & $\begin{array}{l}\text { Affected patients, } \\
n(\%) \\
n=122\end{array}$ & $\begin{array}{l}\text { Affected infusions, } \\
n(\%) \\
n=6676\end{array}$ & AEs/patient & AEs/infusion \\
\hline Total local AEs & 232 & $35(28.7)$ & $181(2.711)$ & 1.902 & 0.035 \\
\hline Infusion/injection site erythema & 77 & $18(14.8)$ & $76(1.138)$ & 0.631 & 0.012 \\
\hline Infusion/injection site pain or discomfort & 57 & $18(14.8)$ & $55(0.824)$ & 0.467 & 0.009 \\
\hline Infusion site swelling & 46 & $4(3.3)$ & $46(0.689)$ & 0.377 & 0.007 \\
\hline Infusion/injection site pruritus & 34 & $10(8.2)$ & $34(0.509)$ & 0.279 & 0.005 \\
\hline Infusion site urticaria & 8 & $3(2.5)$ & $8(0.120)$ & 0.066 & 0.001 \\
\hline Infusion/injection site reaction & 3 & $2(1.6)$ & $3(0.045)$ & 0.025 & $<0.001$ \\
\hline
\end{tabular}

Table 3. Causally related ${ }^{\dagger}$ systemic adverse events (excluding infections) occurring in $\geq 1 \%$ of patients.

\begin{tabular}{|c|c|c|c|c|c|}
\hline Adverse event & AEs, $n$ & Affected patients, n (\%) & Affected infusions, $n$ (\%) & AEs/patient & AEs/infusion \\
\hline Total systemic AEs $\ddagger$ & 165 & $27(22.1)$ & $145(2.172)$ & 1.352 & 0.025 \\
\hline Headache & 70 & $11(9.0)$ & $65(0.974)$ & 0.574 & 0.010 \\
\hline Diarrhea $\ddagger$ & 49 & $4(3.3)$ & $49(0.734)$ & 0.402 & 0.007 \\
\hline Fatigue & 11 & $7(5.7)$ & $11(0.165)$ & 0.090 & 0.002 \\
\hline Nausea & 9 & $5(4.1)$ & $9(0.135)$ & 0.074 & 0.001 \\
\hline Dizziness & 6 & $2(1.6)$ & $6(0.090)$ & 0.049 & $<0.001$ \\
\hline Myalgia & 5 & $3(2.5)$ & $5(0.075)$ & 0.041 & $<0.001$ \\
\hline Migraine & 4 & $2(1.6)$ & $4(0.060)$ & 0.033 & $<0.001$ \\
\hline Somnolence & 3 & $2(1.6)$ & $3(0.045)$ & 0.025 & $<0.001$ \\
\hline \multicolumn{6}{|c|}{$\begin{array}{l}{ }^{\dagger} \text { As assessed by the investigator. } \\
\ddagger 46 \text { AEs (diarrhea events) were reported by } 1 \text { patient with a history of recurrent diarrhea. } \\
\text { AE: Adverse event. }\end{array}$} \\
\hline
\end{tabular}

(0.002/infusion). Of note, one patient in the European study with a medical history of ongoing recurrent diarrhea reported 46/49 of the diarrhea events. For the most frequently reported causally related systemic AE (headache), the median (range) time between onset of the AE and start time of the preceding infusion was 8 (0-169) h and time between AE onset and its resolution was $25.2(0-714) \mathrm{h}$.

\section{Tolerability}

Interruptions or reductions in the infusion rate due to tolerability or AEs were rarely required during treatment with Ig20Gly. Overall, 99.8\% of infusions in both studies were administered without interruption or a reduction in the infusion rate. Moreover, there was no apparent association between infusion volumes and infusion rates per site and the rate of Ig20Gly causally related local AEs (Figures $1 \& 2$ ).

\section{Administration characteristics}

The median (range) number of sites per infusion was 2 (1-5), and the infusion volume was 30.3 (6.4-76.0) $\mathrm{ml}$ per site. The median (range) infusion duration was $0.95(0.2-6.4) \mathrm{h}$, and the maximum infusion rate was 40 (2.5-180.0) $\mathrm{ml} / \mathrm{h}$ per site (Table 4).

\section{Discussion}

Subcutaneous administration of Ig offers a treatment option for patients permitting self-administration at home with fewer systemic AEs than IV administration [6,9,10]. SC administration results in a more gradual absorption and lower peak levels of serum IgG, which likely accounts for the improved systemic tolerability [11]. However, SCIG formulations, in particular those with a low IgG concentration (e.g., 10 or 16\%), have limitations in the volume that can be infused per site $[9,10]$. The development of more highly concentrated SCIG products allows for infusion of the same dose in smaller volumes, thus reducing the number of infusion sites and possibly the frequency of 


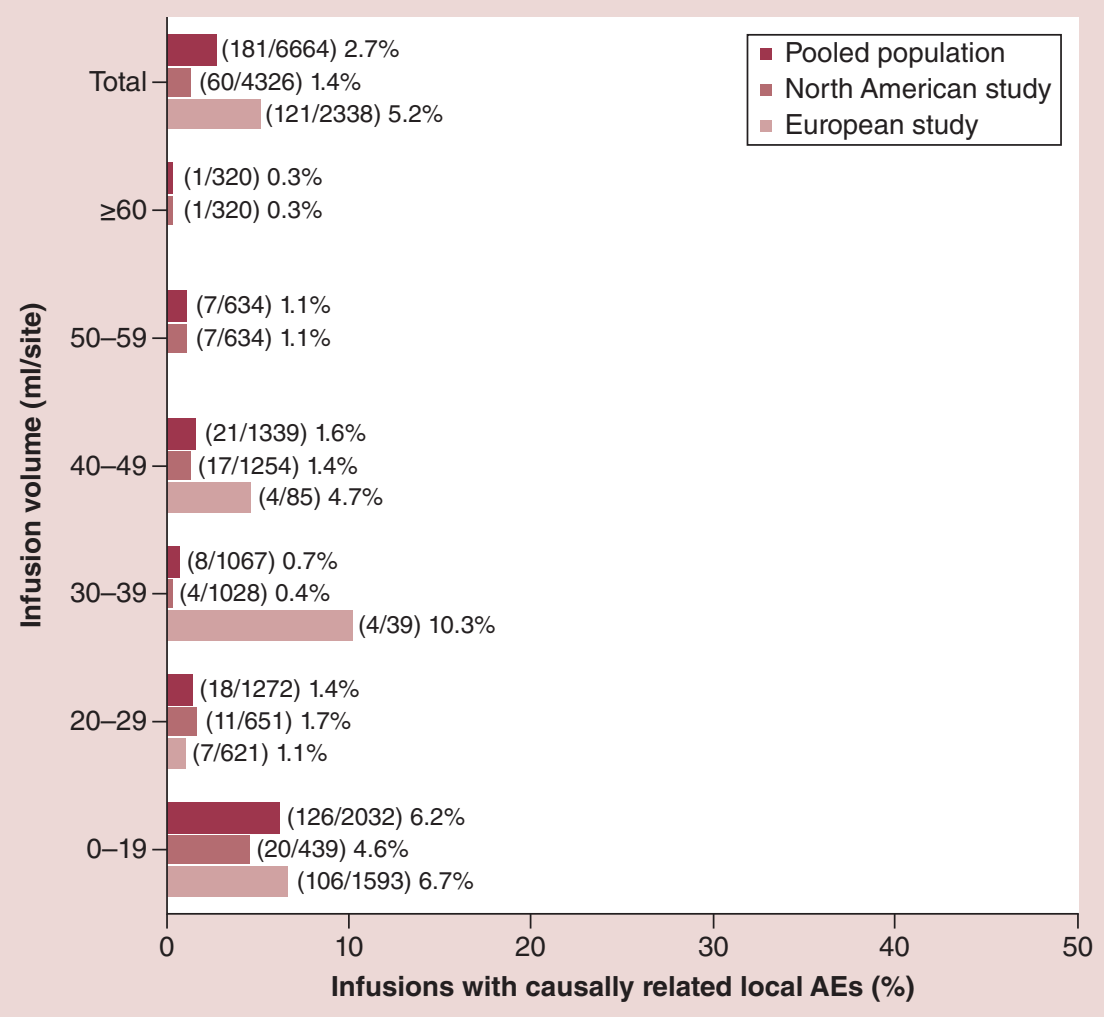

Figure 1. Percentage of infusions with local adverse events by infusion volume ( $\mathrm{ml}$ ) per site for individual studies and pooled data. Portions of individual study data previously reported $[7,8]$.

AE: Adverse event.

Table 4. Ig20Gly administration characteristics.

\begin{tabular}{|c|c|c|c|c|c|c|}
\hline \multirow[t]{2}{*}{ Characteristic } & \multicolumn{2}{|c|}{ European study, $n=48$} & \multicolumn{2}{|c|}{ North American Study, $\mathrm{n}=74$} & \multicolumn{2}{|c|}{ Pooled, $\mathrm{N}=122$} \\
\hline & Infusions, $\mathbf{n}$ & Median, min-max & Infusions, $\mathrm{n}$ & Median, min-max & Infusions, $\mathrm{n}$ & Median, min-max \\
\hline Duration of infusion, $\mathrm{h}$ & 2335 & $0.95(0.3-4.1)$ & 4162 & $0.95(0.2-6.4)$ & 6497 & $0.95(0.2-6.4)$ \\
\hline Sites per infusion, $\mathrm{n}$ & 2338 & $2.0(1-5)$ & 4326 & $2.0(1-4)$ & 6664 & $2.0(1-5)$ \\
\hline Infusion volume, $\mathrm{ml} /$ site & 2338 & $16.6(6.5-48.0)$ & 4326 & $39.5(6.4-76.0)$ & 6664 & $30.25(6.4-76.0)$ \\
\hline $\begin{array}{l}\text { Maximum infusion rate, } \\
\mathrm{ml} / \mathrm{h} / \mathrm{site}\end{array}$ & 2338 & $20.0(2.5-60.0)$ & 4314 & $60.0(4.4-180.0)$ & 6652 & $40.0(2.5-180.0)$ \\
\hline
\end{tabular}

infusions compared with less concentrated SCIG preparations [6]. The 20\% IG product Ig20Gly contains glycine as a stabilizing agent to prevent and minimize IgG dimerization, has a viscosity of $14.4 \mathrm{mPas}$, and has an osmolality of $280-292 \mathrm{mOsm} / \mathrm{kg}$, which is similar to the osmolality of extracellular fluid (280-295 mOsm $/ \mathrm{kg})$. Ig20Gly can be infused at up to $60 \mathrm{ml} / \mathrm{h}$ per site and has been shown to be efficacious and well tolerated in two Phase II/III clinical studies conducted in nine countries in Europe [7] and North America [8]. This pooled safety and tolerability analysis of 6676 infusions of Ig20Gly administered to 122 patients over a median duration of 1 year demonstrated that Ig20Gly was well tolerated in a diverse population of patients with PID.

The number and incidence of local and systemic AEs were low, and no causally related serious or severe AEs were reported with Ig20Gly. As expected, the rate of causally related systemic AEs (0.025/infusion) was lower than that of causally related local AEs (0.035/infusion). Regarding systemic AEs, it is also important to note that causally related, nonserious, systemic AE rates in these studies were substantially lower with SCIG than with IVIG [7,8]. As previously reported $[7,8]$, causally related, nonserious systemic AE rates (the number of AEs divided by the 


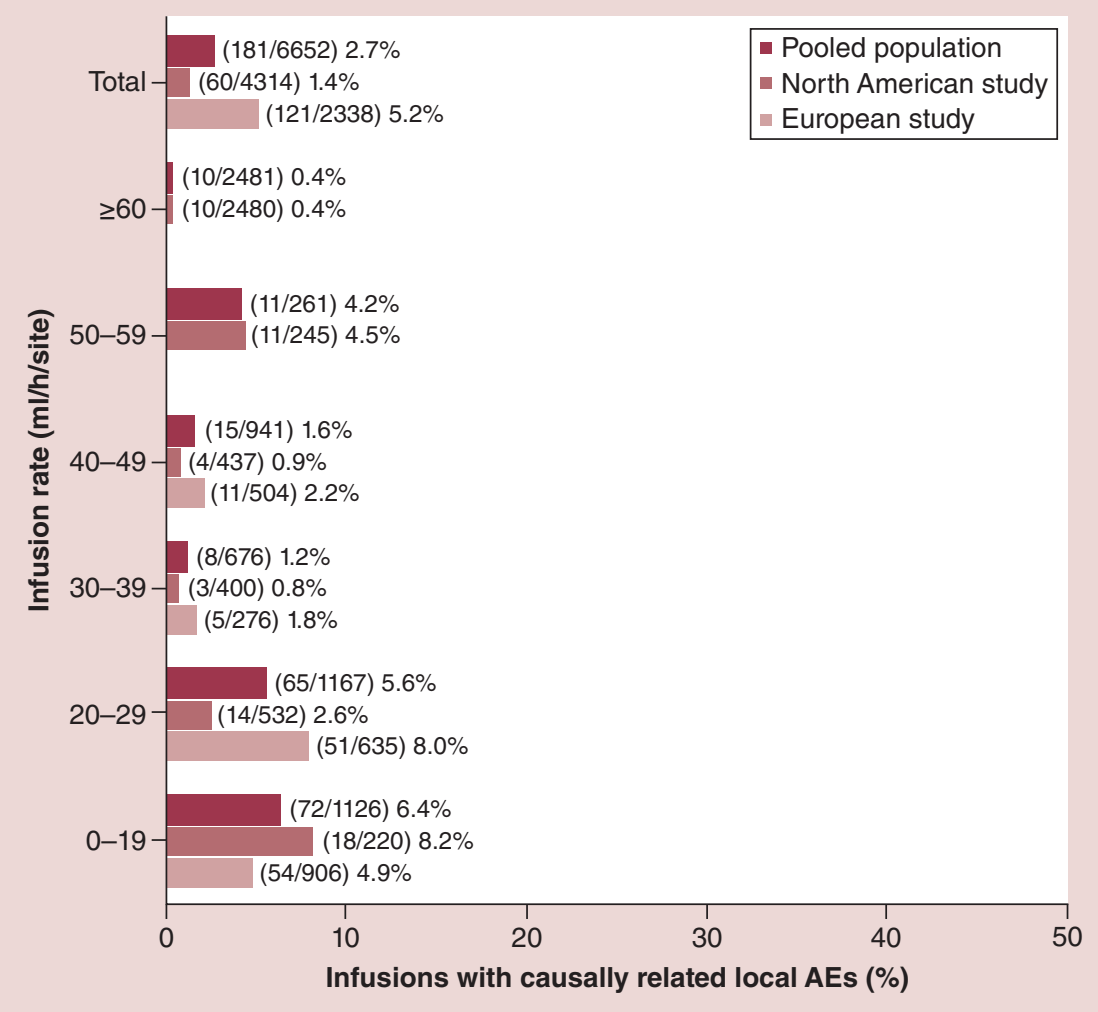

Figure 2. Percentage of infusions with local adverse events by maximum infusion rate per site $(\mathrm{ml} / \mathrm{h} / \mathrm{site})$ for individual studies and pooled data. Individual study data previously reported $[7,8]$.

AE: Adverse event.

number of infusions) with SCIG were approximately 3-fold to 11-fold lower than with IVIG. Furthermore, the frequency of systemic AEs in this pooled analysis may have been higher than expected due to a single patient in the North American study who reported multiple systemic AEs under some administration conditions. Systemic gastrointestinal disorders are common in individuals with PIDs [12]. The use of SCIG over IVIG is preferred in these individuals because systemic AEs, including diarrhea and gastrointestinal-related issues, on SCIG are rare [3]. As such, SCIG is less likely to exacerbate pre-existing gastrointestinal conditions than IVIG.

No treatment-related local AEs were reported in $97.3 \%$ of infusions. Notably, a 13-year-old patient reported 79 of the 232 (34.1\%) local AEs causally related to Ig20Gly in the European study, all of which were mild [7]. This patient was treated with IVIG before study enrollment; however, the patient completed the study as planned and expressed preference for the SCIG Ig20Gly over other IG treatment options at the end of the study.

In further regard to the safety of SCIG, it should be noted that it has previously been reported that there were no instances of hemolysis with SCIG in either study $[7,8]$. Although 12 patients (6 in each study) had temporary declines in hemoglobin of $\geq 2.0 \mathrm{~g} / \mathrm{dl}$, there was no concordance between these declines and other laboratory tests at any time in either study that supported a diagnosis of hemolysis $[7,8]$. Additionally, there were no reported adverse events related to thrombotic risk in these studies.

The favorable tolerability of Ig20Gly infusions allowed the administration of faster infusion rates (median, $40 \mathrm{ml} / \mathrm{h}$ per site) and volumes per site (median, $30 \mathrm{ml}$ per site). The median infusion duration was $<1 \mathrm{~h}$. Overall, $99.8 \%$ of infusions in the two studies did not require interruptions or reductions in the infusion rate. There was no apparent association between infusion volumes and infusion rates per site and the rate of Ig20Gly causally related local AEs.

The combination of relatively large infusion volumes per site, rapid infusion rates and short infusion durations achieved with Ig20Gly is noteworthy. In the North American study, the median of the maximum infusion rate was $60 \mathrm{ml} / \mathrm{h}$ per site and was achieved in more than half of completed Ig20Gly infusions without compromising 
tolerability [8], indicating that the majority of patients can tolerate the maximum recommended infusion rate of $60 \mathrm{ml} / \mathrm{h}$ per site; $71.6 \%$ of patients reached the infusion rate of $\geq 60 \mathrm{ml} / \mathrm{h}$ per site at least once during the North American trial. The median of the maximum infusion rate and volume per site was lower in the earlier European study; however, the ranges in both studies were overlapping, and the median infusion duration and number of sites required were the same $(0.95 \mathrm{~h}$ and 2 sites/infusion, respectively) [7,8]. In general, North American physicians use higher doses than their European counterparts, as evident in the median doses used for patients before entry into the North American trial (IVIG, $146.7 \mathrm{mg} / \mathrm{kg} /$ week; SCIG, $154.0 \mathrm{mg} / \mathrm{kg} /$ week) and the European trial (IVIG, $113.1 \mathrm{mg} / \mathrm{kg} /$ week; SCIG, $114.0 \mathrm{mg} / \mathrm{kg} /$ week). Lower mean doses were also used during the European trial (125 mg/kg/week [7] vs $222 \mathrm{mg} / \mathrm{kg} /$ week in the North American study [8]), which may account for the slower median infusion rates and smaller volumes. This dose difference in the two studies is also attributable to the manner in which the Ig20Gly dose was calculated. In the North American study, a systemic exposure equivalent to previous IVIG 10\% treatment was targeted, and the Ig20Gly dose was adjusted to compensate for the lower bioavailability of SC administration of IgG [13]. In the European study, patients were switched from IVIG to SCIG using a 1:1 dose adjustment ratio, with the aim of maintaining stable $\mathrm{IgG}$ trough levels of $>500 \mathrm{mg} / \mathrm{dl}$.

Safety was assessed in a rigorous, comprehensive manner in the two studies. Investigators were trained in a consistent manner to identify potential AEs, and all patients, including those who received home-based treatment, were evaluated at the study site at regular intervals (i.e,. 8-12 weeks). Patients were given eDiaries in which to record data during and after infusions administered at home, and investigators were required to contact patients 3-5 days after each infusion to ask if an AE had occurred and to ensure all relevant AE data were recorded. Collectively, these patient-centered measures were intended to provide a complete representation of the incidence and severity of AEs, regardless of whether a patient chose to have the infusions administered in the clinic or at home.

In the current analysis of Ig20Gly, the median number of infusion sites used was 2, and the median of the maximum infusion rate and volume per site was $40 \mathrm{ml} / \mathrm{h}$ per site and $30.3 \mathrm{ml}$ per site, respectively; however, patients tolerated a wide range of infusion rates and volumes of $\operatorname{Ig} 20 \mathrm{Gly}$, suggesting that the rate and volume used for IG administration should be individualized for each patient. For the $20 \%$ IG product IgPro20 (Hizentra ${ }^{\circledR}$, CSL Behring AG, Bern, Switzerland) [14], the prescribing information recommends the use of up to eight infusion sites simultaneously, with a maximum volume of $25 \mathrm{ml}$ per site and a maximum rate of $25 \mathrm{ml} / \mathrm{h}$ per site for PID (volumes up to $50 \mathrm{ml}$ per site and rates up to $50 \mathrm{ml} / \mathrm{h}$ per site for chronic inflammatory demyelinating polyneuropathy) [15]. Less concentrated products require a larger volume than more highly concentrated products to administer an equivalent dose of $\mathrm{IgG}$, which is often achieved by using multiple infusion sites. One example of such a product with an IG concentration of $16 \%$ is SUBCUVIA (Baxter Healthcare Ltd, Berkshire, UK), for which the recommended maximum injection volume is $5-15 \mathrm{ml}$ per site and the recommended maximum infusion rate is $20 \mathrm{ml} / \mathrm{h}$, with no limit on the number of infusion sites [16]. Similarly, a product with an IG concentration of $10 \%$, GAMMAGARD LIQUID, requires $\leq 30 \mathrm{ml}$ per site (in up to 8 sites) at an infusion rate of $20-30 \mathrm{ml} / \mathrm{h}$ per site in patients weighing $\geq 40 \mathrm{~kg}$ [17].

One limitation of this analysis is the retrospective combination of data from two studies conducted in geographically separate populations, which may have different clinical practices. Although the study designs were similar in the North American and European trials, the dose of Ig20Gly was calculated differently in the trials, resulting in slightly different infusion parameters between studies. This difference, however, resulted in the ability to gather and compare safety and tolerability parameters across a wider dose range than would otherwise have been possible. In addition, infusion parameters collected during clinical studies may not reflect real-world administration practices.

\section{Conclusion}

The results of this pooled analysis demonstrate that Ig20Gly appears to be safe and well tolerated in a diverse population of patients with PID. Low rates of causally related local and systemic AEs were observed. There was no association between the infusion volume or rate and the rate of casually related local AEs; $99.8 \%$ of infusions were administered without interruption or rate reductions. The favorable tolerability permitted fast infusion rates, large infusion volumes per site and a median infusion duration of $<1 \mathrm{~h}$. The flexibility in selection of infusion rates/volumes per site and favorable tolerability make Ig20Gly a valuable treatment option for adult and pediatric patients with PID. 


\section{Summary points}

- Immunoglobulin (IG) replacement therapy is indicated for the treatment of primary immunodeficiency diseases (PID) with defective antibody production.

- This pooled analysis assessed adverse events (AEs), tolerability and infusion parameters in patients with PID who received the subcutaneous IG $20 \%$ product, Ig20Gly, at volumes of $\leq 60 \mathrm{ml} / \mathrm{site}$ and at rates of $\leq 60 \mathrm{ml} / \mathrm{h} / \mathrm{site}$.

- A total of 122 patients aged 2-83 years received 6676 infusions (mean \pm SD of $54.7 \pm 14.6$ infusions per patient) of Ig20Gly.

- There were no serious or severe AEs related to Ig20Gly treatment reported.

- Thirty-five patients (28.7\%) reported 232 causally related local AEs (frequency rate of 0.035 per infusion) and 27 patients (22.1\%) reported 165 causally related systemic AEs (frequency rates of 0.025 per infusion).

- Overall, $99.8 \%$ of infusions were administered without interruption or a reduction in the infusion rate and there was no apparent association between infusion volume or rate with the rate of Ig20Gly causally related local AEs.

- The median (range) number of sites per infusion was 2 (1-5) and of infusion volume per site was 30.3 (6.4-76.0) $\mathrm{ml}$.

- Subcutaneous immunoglobulin $20 \%$ solution, Ig20Gly, was well tolerated in a broad population of patients across a wide age range with PID from two continents.

\section{Trial registration}

This study reports data from two prospective, open-label clinical trials conducted in North America (registered on www.clinicaltria Is.gov: NCT01218438) and Europe (registered on clinicaltrialsregister.eu: EudraCT \#:2010-019459-23 and www.clinicaltrials.gov, NCT01412385).

\section{Author contributions}

All authors were involved in the conception and design of the study, data generation, data analysis and interpretation, preparation and writing of the manuscript, critical review of the manuscript and approved the final version of the manuscript. D Suez, G Krivan, S Jolles, M Stein, S Gupta, K Paris, PM van Hagen and N Brodszki were investigators who enrolled patients in the North American and European trials. W Engl was the statistician, and $\mathrm{H}$ Leibl, B McCoy, and L Yel were clinical leads involved in each trial.

\section{Acknowledgments}

The authors would like to thank the investigators who enrolled patients in the North American and European trials and the patients and their parents/caregivers for their participation in these trials.

Financial \& competing interests disclosure

The clinical studies were funded by Baxalta, a member of the Takeda group of companies. D Suez, G Krivan, and PM van Hagen, have no competing interests. $S$ Jolles has participated in advisory boards, trials, projects, and has been a speaker with Baxalta, CSL Behring, Shire, Thermofisher, Swedish Orphan Biovitrum Pharming, Biotest, Binding Site, Grifols, BPL, Octapharma, LFB, GSK, Weatherden, Zarodex, Sanofi and UCB Pharma. S Gupta is an investigator for Baxalta/Shire/Takeda, Octapharma and ProMatic; served on an advisory board for Octapharma; and received investigator initiated grants from Baxalta/Shire and Octapharm. M Stein is a Shire investigator and speaker and is an investigator for Grifols, BPL, Therapure, Prometic, Green Cross and Kedrion. $\mathrm{K}$ Paris has served on advisory boards and as a speaker for Shire, and on advisory boards for Grifols. N Brodszki has served on advisory boards and as a speaker or participated in projects with CSL Behring, Baxter/Baxalta/Shire, Octapharma, and Meda. W Engl, H Leibl, B McCoy, and L Yel are employees of the Takeda group of companies. The authors have no other relevant affiliations or financial involvement with any organization or entity with a financial interest in or financial conflict with the subject matter or materials discussed in the manuscript apart from those disclosed.

Scientific writing support for this manuscript was provided by B John, and LMK Callan, at C4 MedSolutions, LLC (Yardley, PA, USA), a CHC Group company, and was funded by Shire, a member of the Takeda group of companies.

Ethical conduct of research

This is a pooled analysis of two prospective, open-label clinical trials in Europe (ClinicalTrials.gov NCT01412385; Borte M et al. Clin. Exp. Immunol. 187[1], 146-159 [2017]) and North America (ClinicalTrials.gov NCT01218438; Suez D, et al. J. Clin. Immunol. 36[7], 700-712 [2016]). Both studies were performed in accordance with the Declaration of Helsinki and the international standards of Good Clinical Practice. Patients were enrolled at 15 sites in the USA and Canada for the North American study, and at 16 sites in seven countries for the European study. Written informed consent was obtained from all patients before undergoing any study procedure. 


\section{Data sharing statement}

The authors certify that this manuscript reports the secondary analysis of clinical trial data that have been shared with them, and that the use of this shared data is in accordance with the terms (if any) agreed upon their receipt. The source of this data is: NCT01218438; NCT01412385. The datasets, including redacted study protocol, redacted statistical analysis plan, and individual participant data behind the results reported in this article, will be available 3 months after manuscript publication to researchers who provide a methodologically sound proposal after de-identification, in compliance with applicable privacy laws, data protection, and requirements for consent and anonymization. Data requests should follow the process outlined in the Data Sharing section of the Sponsor's website: http://www.shiretrials.com/en/our-commitment-to-transparency/data-sharing-with-researchers and should be directed to clinicaltrialdata@shire.com

\section{References}

Papers of special note have been highlighted as: $\bullet$ of interest; $\bullet \bullet$ of considerable interest

1. Bousfiha A, Jeddane L, Picard C et al. The 2017 IUIS phenotypic classification for primary immunodeficiencies. J. Clin. Immunol. 38(1), 129-143 (2018).

- This review provides a detailed overview of the single-gene inborn errors of immunity and their phenotypic classifications.

2. Orange JS, Hossny EM, Weiler CR et al. Use of intravenous immunoglobulin in human disease: a review of evidence by members of the Primary Immunodeficiency Committee of the American Academy of Allergy, Asthma and Immunology. J. Allergy Clin. Immunol. 117(4 Suppl.), S525-553 (2006).

3. Stiehm ER. Adverse effects of human immunoglobulin therapy. Transfus. Med. Rev. 27(3), 171-178 (2013).

- This review provides an overview of the adverse effects of intravenously and subcutaneously administered human immunoglobulins.

4. Gardulf A, Nicolay U, Math D et al. Children and adults with primary antibody deficiencies gain quality of life by subcutaneous IgG self-infusions at home. J. Allergy Clin. Immunol. 114(4), 936-942 (2004).

5. Nicolay U, Kiessling P, Berger M et al. Health-related quality of life and treatment satisfaction in North American patients with primary immunedeficiency diseases receiving subcutaneous IgG self-infusions at home. J. Clin. Immunol. 26(1), 65-72 (2006).

6. Jolles S, Orange JS, Gardulf A et al. Current treatment options with immunoglobulin G for the individualization of care in patients with primary immunodeficiency disease. Clin. Exp. Immunol. 179(2), 146-160 (2015).

-. This narrative review describes the goals of immunoglobulin replacement therapy, therapeutic options and therapy selection, and the management of adverse events associated with treatment.

7. Borte M, Krivan G, Derfalvi B et al. Efficacy, safety, tolerability and pharmacokinetics of a novel human immune globulin subcutaneous, 20\%: a Phase 2/3 study in Europe in patients with primary immunodeficiencies. Clin. Exp. Immunol. 187(1), 146-159 (2017).

-. This prospective non-controlled clinical trial in patients with primary immunodeficiency diseases conducted in Europe demonstrated that the subcutaneous immunoglobulin $20 \%$ product, Ig20Gly, was effective and well tolerated.

8. Suez D, Stein M, Gupta S et al. Efficacy, safety, and pharmacokinetics of a novel human immune globulin subcutaneous, $20 \%$ in patients with primary immunodeficiency diseases in North America. J. Clin. Immunol. 36(7), 700-712 (2016).

-. This prospective open-label clinical trial in patients with primary immunodeficiency diseases conducted in North America demonstrated that the subcutaneous immunoglobulin $20 \%$ product, Ig20Gly, was effective and well tolerated.

9. Wasserman RL. Progress in gammaglobulin therapy for immunodeficiency: from subcutaneous to intravenous infusions and back again. J. Clin. Immunol. 32(6), 1153-1164 (2012).

10. Melamed I, Testori A, Spirer Z. Subcutaneous immunoglobulins: product characteristics and their role in primary immunodeficiency disease. Int. Rev. Immunol. 31(6), 451-461 (2012).

11. Kobrynski L. Subcutaneous immunoglobulin therapy: a new option for patients with primary immunodeficiency diseases. Biologics 6, 277-287 (2012).

12. Agarwal S, Mayer L. Diagnosis and treatment of gastrointestinal disorders in patients with primary immunodeficiency. Clin. Gastroenterol. Hepatol. 11(9), 1050-1063 (2013).

13. Berger M, Jolles S, Orange JS, Sleasman JW. Bioavailability of IgG administered by the subcutaneous route. J. Clin. Immunol. 33(5), 984-990 (2013).

14. Hagan JB, Fasano MB, Spector S et al. Efficacy and safety of a new $20 \%$ immunoglobulin preparation for subcutaneous administration, IgPro20, in patients with primary immunodeficiency. J. Clin. Immunol. 30(5), 734-745 (2010).

15. Hizentra (immune globulin subcutaneous [human], 20\% liquid). Full Prescribing Information, CSL Behring AG, Bern, Switzerland (2016).

16. SUBCUVIA $160 \mathrm{~g} / 1$ solution for injection (human normal immunoglobulin). Summary of Product Characteristics, Baxalta UK Ltd, Berkshire, UK (2018).

17. Gammagard Liquid (immune globulin infusion [human], 10\% solution). Full Prescribing Information, Baxalta US Inc., CA, USA (2016). 
Research Article Suez, Kriván, Jolles et al. 